\title{
La finzione letteraria della sicilianità in Camilleri L'esempio de La voce del violino
}

Domenica Elisa Cicala

Alpen-Adria Universität Klagenfurt domenica.cicala@uni-klu.ac.at

Riassunto: Il presente contributo si propone un'indagine ermeneutica volta a evidenziare il modo in cui nel giallo La voce del violino prende forma l'immagine della Sicilia e a far emergere gli aspetti in cui può rintracciarsi la rappresentazione letteraria della sicilianità, intesa come summa di elementi peculiari dell'isola e dei suoi abitanti.

Parole chiavi: Camilleri. Rappresentazione letteraria. Sicilia. Cultura. Società.

Abstract: Through a hermeneutical investigation this paper aims to examine the image of Sicily in La voce del violino and to trace aspects of the literary representation of the sicilianity, understood as the sum of particular factors of the island and its inhabitants.

Key words: Camilleri. Literary representation. Sicily. Culture. Society.

\section{Introduzione}

Dopo La forma dell'acqua (1994), Il cane di terracotta (1996) e Il ladro di merendine (1996), nel 1997 esce presso Sellerio La voce del violino e, a detta dell'autore, inizia a rendersi percepibile tra il pubblico dei suoi lettori il cosiddetto "caso Camilleri":

[...] quando pubblicai «La voce del violino» e andai per librerie a presentarlo, notai una novità: che il pubblico non era più solo dai 40 in su, cominciavano a esserci giovani di 20 anni, magari con l'orecchino, 
La finzione letteraria della sicilianità in Camilleri - L'esempio de La voce del violino

Domenica Elisa Cicala

che ti facevano osservazioni intelligentissime $[\ldots]$. Il caso $[\ldots]$ cominciava a farsi percepire (SORGI 2000, p. 76). ${ }^{1}$

All'enorme successo della serie di gialli con protagonista il commissario Montalbano può aver contribuito la scelta di fare della sicilianità un ingrediente fondamentale dell'impasto narrativo, affidando al testo un tono di plausibilità e verosimiglianza. ${ }^{2}$ In un microcosmo letterario che trae ispirazione dalla realtà, Camilleri tratteggia i connotati di un'umanità costretta ad adattarsi ai luoghi, ad affrontare irrisolti problemi sociali, a interpretare modalità espressive ibride, ad assumere un atteggiamento sereno e rassegnato davanti alla vita, magari gustandone i sapori e i lati migliori. È proprio la sicilianità di un contesto dai caratteri molteplici e contrastanti a dare risalto, tra gli altri personaggi, in particolare al protagonista che, da figura centrale, si fa veicolo di trasmissione di valori antropologici della terra da cui proviene e offre un'immagine nitida della società in cui vive. Camilleri, dunque,

\footnotetext{
${ }^{1}$ Sul "caso" Camilleri cfr. Buttitta (ed.), 2004; Capecchi, 2000, pp. 9-11; Palumbo, 2005, pp. 11-12; Sorgi, 2000, pp. 13-21. Sull'autore e la sua opera cfr. Bonina, 2007. Del giallo è stata realizzata nel 1999 una riduzione cinematografica con la regia di A. Sironi. Tra le ragioni del successo della serie televisiva con protagonista il commissario Montalbano Privitera menziona la seguente: "Ma è soprattutto merito del regista saper rendere la sicilianità della scrittura e del pensiero per proiettarlo nel paesaggio [...]" (2009, p. 116).

${ }^{2}$ Sul criterio di verosimiglianza nei gialli di Camilleri cfr. Demontis, 2001, pp. 68-69, p. 72. Sul tema della verità come spunto per la fantasia di Camilleri si legge in Sorgi, 2000, p. 74: “[...] io le storie non me le so inventare di sana pianta; ho bisogno di una spinta di verità"; ivi, p. 103: "[...] io ho sempre bisogno di uno spunto reale, un momento di verità". A riguardo scrive Vitale, 2001, p. 120: "Alla fine di ogni opera il Maestro si affanna a sostenere che fatti, personaggi e situazioni sono stati inventati. Non credetegli! Fatti e personaggi sono esistiti realmente, esistono realmente e continueranno realmente ad esistere. Magari ci potranno essere state delle fusioni; di due o più persone esistenti ne è stata fatta una sola, ma tipi e caratteri non sono assolutamente inventati, ma captati dall'occhio attento ed osservatore del Maestro".
} 
definendosi "uno scrittore italiano nato in Sicilia" (SORGI 2004, p. 42), affonda le radici della sua vena creativa nella sua isola natale e la rende cassa di risonanza di una ricchezza umana e culturale. Vediamo in che modo.

\section{L'ambientazione topografica}

Il primo elemento tangibile che riconduce i gialli di Camilleri alla Sicilia consiste nella scelta dell'isola come scenario d'ambientazione, ricco di sfumature tanto contraddittorie quanto affascinanti, che contribuiscono a renderlo non solo sfondo, bensì a volte vero protagonista. Le vicende del commissario Salvo Montalbano sono ambientate a Vigàta, uno spazio fittizio che nella mente dell'autore richiama il suo luogo natale di Porto Empedocle, in provincia di Agrigento, città resa riconoscibile nelle descrizioni del centro storico e della zona del Rabato e chiamata pirandellianamente Montelusa. ${ }^{3}$ Più precisamente, come Camilleri dichiara in un'intervista, l'idea di Vigàta gli è nata dal ricordo della piazzetta S. Francesco di Agrigento, poiché l'entrata al liceo da lui frequentato era proprio su questa piccola piazza, accanto all'omonima chiesa, davanti alla quale tutti gli studenti si incontravano prima delle lezioni e parlavano dei fatti successi nelle zone di appartenenza:

L'idea di Vigàta come paese a geometria variabile nasce dalla memoria che ho di quella piazzetta, dove mi era possibile sapere cose che capitavano in altre parti della provincia e che, inevitabilmente, diventavano mie. Vigàta è quella piazzetta [...]. (FERLITA 2003, p. 15)

Tale immagine di Vigàta come luogo d'incontro di persone e scambio di informazioni può essere rintracciata ne La voce del violino nella presenza,

\footnotetext{
${ }^{3}$ Sull'influenza di Pirandello su Camilleri, tra gli altri, cfr. Capecchi, 2000, pp. 11-16 e Pistelli, 2003, pp. 44-53.
} 
accanto ai siciliani, di personaggi che provengono da diverse parti d'Italia e accentuano i connotati di una realtà piccola e provinciale, ma aperta ai contatti con l'esterno. Se, infatti, la fidanzata di Montalbano, Livia Burlando, è di Boccadasse in provincia di Genova, il giudice Nicolò Tommaseo è veneziano, il questore Luca Bonetti-Alderighi, invece, bergamasco, il capo della Scientifica il dottor Arquà è stato trasferito da Firenze, la vittima Michela Licalzi e l'assassino, il suo amante Guido Serravalle, sono bolognesi e, tra i picciotti che lavorano la terra nella tenuta della sorella di Mimì, c'è anche Ennst, un ventenne tedesco che si trova lì di passaggio e che spera di fermarsi in Sicilia per altri tre mesi.

Dall'isola non vorrebbe mai allontanarsi Salvo Montalbano, strettamente ancorato al suo universo. Del territorio siciliano il commissario ama soprattutto l'asprezza dell'entroterra, tanto che per poter osservare i paesaggi brulli e riarsi è solito tralasciare le strade principali e percorrere vie alternative, stradine spesso in condizioni disagiate, trazzere terremotate sulle quali "persino le capre nutrivano qualche perplessità a metterci sopra una sola delle quattro zampe di cui disponevano" (p. 100).

Quella però era la Sicilia che piaceva al commissario, aspra, di scarso verde, sulla quale pareva (ed era) impossibile campare e dove ancora c'era qualcuno, ma sempre più raro, con gambali, coppola e fucile in spalla, che lo salutava da sopra la mula portandosi due dita alla pampèra (p. 101). ${ }^{4}$

Preferendo la solitudine di spazi abbandonati, in questo giallo Montalbano cerca il contatto con la natura e, sedutosi su un ramo di un albero d'ulivo, "un gigantesco olivo saraceno, di quelli storti e contorti che strisciano sulla terra

\footnotetext{
${ }^{4}$ All'amore di Montalbano verso l'arida Sicilia dell'entroterra si fa riferimento anche in altri gialli, tra cui si vedano Il cane di terracotta, p. 72 e L'odore della notte, p. 111.
} 
come serpenti prima di alzarsi verso il cielo" (p. 137), ragiona sui fatti successi. Uno stimolo a riordinare le idee e a pensare su quanto accade gli è anche offerto dall'osservazione del mare che rappresenta per lui un mezzo di riflessione, influenza il suo stato d'animo e comunica sensazioni. ${ }^{5}$

In un evidente connubio tra fantasia e realtà, accanto a luoghi inventati dall'autore, vengono menzionate nel testo località realmente esistenti, come, ad esempio, Floridia, vicino Siracusa (p. 10), Palermo e l'aeroporto di Punta Raisi (p. 38), si fa riferimento a Catania e a tre località in provincia di Enna, e cioè Calascibetta, Gagliano e Troina (p. 100) e si accenna alle località di Aragona e Comitini, in provincia di Agrigento (p. 165). ${ }^{6} \mathrm{Ma}$ a differenza di Giorgio Scerbanenco e Loriano Machiavelli che, rispettivamente, in Traditori di tutti e I sotterranei di Bologna focalizzano la loro attenzione il primo su Milano e il secondo su Bologna e, descrivendo l'atmosfera cupa e fosca delle due grandi città, affidano al contesto sociale e topografico un ruolo determinante per capire la trama dei loro gialli, Camilleri colloca le proprie storie in uno spazio quasi stilizzato che fa da cornice a una vicenda che potrebbe svolgersi anche altrove. In accordo con Crovi si può, perciò, affermare che:

\footnotetext{
Andrea Camilleri ha saputo infondere nelle sue storie i colori e gli odori di una terra forte come la Sicilia, un'isola che l'autore ci racconta in maniera speculare e autonoma rispetto a illustri predecessori come Pirandello, Vittorini, Tomasi di Lampedusa, Brancati, Sciascia. [...] è la Sicilia a tutto tondo nei suoi paesaggi e nei suoi personaggi a diventare oggetto dell'indagine di Camilleri. (CROVI 2002, p. 186)
}

\footnotetext{
${ }^{5} \mathrm{Ne}$ La voce del violino si legge, ad esempio: "Dalla doccia ne uscì con la pelle colore aragosta: per equilibrare la sensazione di freddo provata alla vista del mare aveva abusato d'acqua bollente" (p. 11).

${ }^{6}$ Per una dettagliata descrizione dei luoghi presenti nel giallo in esame cfr. Clausi (et al.), 2007, pp. 81-102; per un elenco dei luoghi di Montalbano cfr. Vitale, 2001, pp. 105-107.
} 
Ritornando con il ricordo nei suoi luoghi d'origine, Camilleri li rende dei generatori narrativi e mediante una rielaborazione creativa li fa rivivere sulla pagina, in un evidente miscuglio tra realtà e finzione letteraria.

\section{La rappresentazione dei problemi sociali}

Senza tacere la presenza di piaghe sociali, corruzione e criminalità, Camilleri non rinuncia all'uso di un tono ironico per indurre il lettore a riflettere e ne La voce del violino, pur non affidandole nello sviluppo della diegesi un ruolo determinante, menziona la presenza dell'organizzazione mafiosa che, a causa dell'arresto e del pentimento di vari membri, vive un momento di sorprendente stasi:

[...] da due mesi non capitava niente di sostanzioso. Per esempio, le famiglie Cuffaro e Sinagra, le due cosche che si contendevano il territorio e che erano solite far ritrovare, con bella regolarità, un morto ammazzato al mese (una volta uno dei Cuffaro e la volta appresso uno dei Sinagra), parevano da qualche tempo avere perso l'entusiasmo. E questo da quando Giosuè Cuffaro, arrestato e fulmineamente pentito dei suoi delitti, aveva mandato in galera Peppuccio Sinagra il quale, arrestato e fulmineamente pentito dei suoi delitti, aveva fatto chiudere in càrzaro Antonio Smecca, cugino dei Cuffaro, il quale, fulmineamente pentito dei suoi delitti, aveva inguaiato Cicco Lo Carmine, dei Sinagra, il quale... (pp. 112-113)

Mediante la ripetizione del termine "fulmineamente" l'autore vuole ribadire la tempestività con cui gli arrestati diventano pentiti pronti a collaborare con la

\footnotetext{
${ }^{7}$ Sull'importanza del ricordo e della memoria nella costruzione fittizia dei luoghi in cui ambientare le vicende afferma Pistelli, 2003, p. 109: "Nonostante la Sicilia nella quale egli si muove possa infatti a volte apparire più che una terra reale un luogo della memoria, un'isola filtrata attraverso ricordi e sensazioni di gioventù, se non in alcuni casi addirittura reinventata, forse proprio per questo, per sfuggire a possibili slanci lirici se non retorici ecco irrompere spesso, in una sorta di utile controcampo, squarci di drammatica realtà contemporanea". A tal proposito si veda, inoltre, Palumbo, 2005, p. 73: "[...] la Vigàta che compare nei romanzi non è la Vigàta (o Porto Empedocle) di oggi, [...] ma quella della memoria dello scrittore".
} 
giustizia e, concludendo la frase con i puntini di sospensione, rende evidente il ripetersi prevedibile del loro comportamento. In seguito a tali arresti eccellenti, mentre il questore afferma orgoglioso in una conferenza stampa che "i numeri uno sono tutti in carcere!" (p. 113), Montalbano, invece, manifesta la consapevolezza che "quelli a cinque stelle stanno al posto loro" (ibid.) e continuano ad agire incontrastati sul territorio. ${ }^{8}$ Nel giallo in esame $i$ mafiosi hanno la possibilità di far scoppiare uno scandalo in questura: per mezzo di un video girato da non altrimenti noti cacciatori mafiosi, amici dell'avvocato Guttadauro, viene fatta luce sull'uccisione di Maurizio Di Blasi, colpito a morte dai colleghi della Mobile, e viene dimostrato come il giovane li avesse minacciati con una delle sue scarpe, erroneamente considerata un'arma dai colleghi di Montalbano. In possesso di questa prova schiacciante, tramite l'avvocato, "penalista discusso, difensore di tutti i mafiosi della provincia e macari di fora provincia" (p. 127), i mafiosi si mettono in contatto con Montalbano che, però, è in grado di cogliere gli indizi, comprende il gioco di Guttadauro e non permette che "il capo della Mobile di Montelusa sia ostaggio della mafia, sia ricattabile" (p. 154). Grazie al suo intuito capisce che occorre giocare d'astuzia e riesce a neutralizzare sul nascere il tentativo mafioso di strumentalizzare un omicidio, relegando così la presenza della mafia sullo sfondo dell'indagine.

Alla straordinaria capacità del commissario di impedire un'eventuale interferenza mafiosa si affianca l'atteggiamento di collaborazione assunto dai siciliani. Quello di Michela Licalzi non è un omicidio legato ad interessi

\footnotetext{
${ }^{8}$ Per approfondire l'opinione di Camilleri sull'argomento "mafia" cfr. Lodato, 2002, pp. 269-312. Sul ruolo che la mafia svolge nei gialli di Camilleri scrive Pistelli, 2003, p. 86: "Parlando di storie poliziesche ambientate in Sicilia, non può essere eluso il tema della mafia e la prima cosa che sorprende nelle vicende di Montalbano è che invece se ne parli solo in modo marginale". Sul fatto che la mafia faccia parte della realtà camilleriana, ma che non si imponga come fenomeno dominante sostiene Meter, 2000, p. 151: “[...] leuchtet ein, dass Camilleris Texte keine MafiaRomane sein können. Immer wieder erscheinen die mafiösen Umtriebe als klischeehaft, vorhersehbar und intellektuell wenig herausfordernd. So ist die Mafia stets gegenwärtig, Teil des sizilianischen Alltags, doch nicht ein alles dominierendes Phänomen”.
} 
mafiosi, ma coloro che hanno visto la vittima prima dell'omicidio, ossia l'addetto a un distributore di benzina, il proprietario di un bar-ristorante e un testimone, contraddicendo ogni stereotipato codice di silenzio, riferiscono alla polizia ciò che hanno visto. In tale aspetto può rintracciarsi quel senso di passione civile a cui Camilleri fa riferimento nel seguente passaggio:

\footnotetext{
La mia Sicilia non è la terra sonnolenta e rassegnata che in tanti hanno narrata (non Sciascia, non Pirandello): essa, semmai, nei miei libri è costantemente in movimento, in rivolta contro qualcosa o qualcuno. Che poi io racconti queste vicende in modo ironico o che possa far scivolare il lettore in un'aperta risata, questo non significa né mancanza di passione e ancor meno assenza di passione civile: è un modo, appunto civile, di esporre dei problemi molto seri. (PISTELLI 2003, p. 167)
}

Tra i vari problemi a cui si accenna nel testo, appare all'ordine del giorno il rischio di subire uno scippo, motivo per cui i siciliani che conoscevano Michela Licalzi temevano per lei, dal momento che se ne andava in giro con una sacca sempre piena di gioielli. Inoltre, in una regione con un alto tasso di disoccupazione, hanno luogo le proteste di un gruppo di trentacinque operai di un cementificio che, messi in cassa integrazione, manifestano e creano disordini (p. 66). Sotto gli occhi di tutti appare anche la corruzione e la piaga dell'abusivismo edilizio, come si legge nella seguente frase: "L'albergo stava a metà strata tra Vigàta e Montelusa, era stato costruito proprio a ridosso di uno dei templi più belli del mondo, alla faccia di sovrintendenze artistiche, vincoli paesaggistici e piani regolatori" (p. 191). Il milieu ristretto e provinciale nell'ambito del quale si svolge la vicenda diviene, quindi, luogo di osservazione sociale, indagato e ispezionato con perizia. Tuttavia, se l'ambientazione del delitto è siciliana, i problemi sociali menzionati non appaiono come elementi strutturali allo svolgimento della vicenda, che ha come assassino il bolognese Guido Serravalle e che presenta come movente i 
suoi debiti di gioco. Pertanto, mentre leggendo Il giorno della civetta di Sciascia si è indotti a riflettere sulla scelta dell'autore di inserire un investigatore non siciliano e a chiedersi se il capitano Bellodi possa essere d'aiuto in una realtà siciliana che non conosce fino in fondo, al contrario ne La voce del violino si è portati a pensare che il male può venire anche da fuori della Sicilia, in quanto a non essere siciliano è proprio l'assassino. La storia ambientata nell'isola non diventa, perciò, pretesto per rappresentare in maniera esplicita la realtà sociale siciliana e le sue piaghe sociali, ma appare piuttosto un'occasione con cui, pur sfiorandone i problemi, è possibile mostrare un volto diverso, ma anch'esso plausibile, di una terra che ha voglia di riscattarsi. ${ }^{9}$

\section{Il linguaggio}

Non trascurabili come veicolo di espressione della sicilianità appaiono le scelte linguistiche di Camilleri che, intrecciando all'italiano standard termini dialettali, fa del bilinguismo la cifra caratterizzante la narrazione e mediante la presenza di termini storpiati conferisce al discorso un tono di vivace sperimentalismo. ${ }^{10}$ Influenzato dalla propria esperienza di regista e insegnante di teatro, ${ }^{11}$ l'autore realizza un impasto linguistico sui generis, mettendo, in

\footnotetext{
${ }^{9}$ Sul volto positivo della Sicilia camilleriana afferma Capecchi, 2000, p. 10: “[...] una Sicilia in movimento, capace di reagire alle ingiustizie e alle prepotenze, ponendo il lettore di fronte a situazioni in cui i personaggi positivi riescono generalmente ad avere la meglio su quelli negativi". A riguardo scrive Crovi, 2002, p. 183: "Camilleri è riuscito a dare una nuova immagine della terra di Sicilia, pur avendo ben presenti modelli come quelli di Sciascia e Pirandello". Sull'influenza di Sciascia su Camilleri si vedano: Capecchi, 2000, pp. 30-32; Demontis, 2001, pp. 158-169; Pistelli, 2003, pp. 53-61. In particolare, quest'ultimo afferma che: "Pirandello e Sciascia [...] si impongono $[\ldots]$ come numi tutelari, pilastri portanti, su cui poggia la più autentica vena narrativa e sicilianità camilleriana” (p. 57). Sulla sicilianità o sicilitudine di e per Camilleri cfr. Sorgi, 2000, pp. 22-61.

${ }^{10}$ Per facilitare la comprensione del linguaggio camilleriano è stato realizzato il seguente dizionario: Bonfiglio, 2002. Per approfondire aspetti del linguaggio di Camilleri, tra i numerosi contributi, si vedano: Capecchi, 2000, Cerrato, 2012, pp. 87-119; pp. 85-98; Demontis, 2001, pp. 9-62; Palumbo, 2005, pp. 99-114; Pistelli, 2003, pp. 14-26; Sorgi, 2000, pp. 117-122.

${ }^{11}$ Sull'importanza dell'esperienza teatrale nella narrativa di Camilleri scrive Palumbo, 2005, p. 23: "Il teatro è l'anima del percorso letterario di Camilleri. Nella palestra del teatro e in quella
} 
particolare, sulla bocca dell'agente Catarella forme linguistiche ibride che, volte a far sorridere, richiamano le modalità espressive di Ulisse, protagonista del dramma satiresco di Euripide Il Ciclope nella traduzione in dialetto realizzata da Pirandello nel 1918. Secondo Salvatore Ferlita un ruolo determinante nella creazione linguistica di Camilleri ha svolto la sua attività di regista per la messa in scena della versione pirandelliana dell'opera euripidea, caratterizzata dall'uso di una lingua consistente in una specie di caricatura dell'italiano sicilianizzato che crea situazioni comiche. ${ }^{12}$ Accanto alle parole di Ulisse Ferlita ne riporta alcune di Catarella per dimostrare la loro comunanza linguistica e concludere che: "[...] l'appuntato Catarella, in quanto esilarante e irresistibile invenzione linguistica, è una diretta filiazione dell'Ulisse di Euripide sub specie dialettale" (FERLITA 2003, p. 25). Personaggio preso dalla realtà, Catarella si esprime in un registro che si adegua al contesto e alla gravità della situazione, come si evince dal passo che segue:

\footnotetext{
«Domando pirdonanza e compressione, dottori». Ahi. Domandava perdono e comprensione. Montalbano appizzò le orecchie, se il cosiddetto italiano di Catarella diventava cerimonioso e pomposo, veniva a significare che la quistione non era leggera (p. 10).
}

Per avere con lui "un dialogo entro limiti tollerabili di delirio" (ibid.) Montalbano adotta il suo stesso linguaggio maccheronico e storpia anche lui le parole, non senza qualche sorpresa. A minare l'integrità linguistica dell'agente

dell'esperienza televisiva il Camilleri scrittore mette a fuoco - e da parte - i procedimenti del congegno narrativo".

${ }^{12}$ A proposito del linguaggio dei personaggi de Il Ciclope pirandelliano scrive Ferlita, 2003, pp. $24-$ 25: "[...] da un lato, il dialetto del Ciclope, che è una specie di massacro con la sua parlata splendidamente contadina; dall'altro, il dialetto di Ulisse, che si muove in un livello di eleganza rusticana. L'eroe omerico, infatti, assomiglia a un villano impettito che voglia darsi delle arie colorando il suo discorso di screziature borghesi, e assumendo in certi casi quasi un accento di superiorità, denunziato soprattutto dalla fonetica italianizzante. Un dialetto borghese, quello di Ulisse, che attinge abbondantemente alla lingua comune e che, per questo motivo, provoca frizioni umoristiche e grottesche [...]". 
è, infatti, in questo giallo la frequenza del corso di "informaticcia" (p. 37) che, costringendolo a esprimersi in italiano, lo porta a trasformarsi e a correggere il commissario in questo modo:

\author{
«Come va il corso d'informaticcia?». \\ «Bene, dottore. Però si dice informatica, dottore». \\ Montalbano lo taliò strammato mentre quello nisciva. Glielo stavano \\ corrompendo, a Catarella" (p. 146).
}

In questo caso la correttezza linguistica diventa paradossalmente sinonimo di corruzione ed è vista da Montalbano addirittura con sospetto.

A parte la parentesi costituita dall'idioletto di Catarella, nella finzione letteraria del mezzo narrativo il linguaggio mantiene i connotati di autenticità e rispecchia nei termini dialettali caratteristiche tipiche della varietà regionale siciliana. Analizzando nel dettaglio alcuni aspetti, ne $L a$ voce del violino a proposito del vocalismo s'incontrano la metafonia (ad esempio, a p. 21 si usa il termine "fimmina" al posto di "femmina") e il processo di dissimilazione, per cui le vocali "e" e "o" vengono ridotte a "i" e "u" (ad esempio, invece di "sole" si usa "suli" a p. 23), mentre in genere le vocali "u" a fine parola, tipiche del siciliano, in Camilleri vengono trasformate quasi sempre in "o". Riguardo al consonantismo sono presenti vocaboli che differiscono dall'italiano standard per l'uso delle geminate (come, ad esempio, "matinata" a p. 9 e "fastiddio"a p. 30), si riscontrano casi di raddoppiamento fonosintattico (come in "quassotto" a p. 27) ed è usata la trascrizione della pronuncia costrittiva del nesso "dr" (ad esempio, nei vocaboli "gaddrina" a p. 12, "picciliddro" a p. 38 e "ciriveddro" a p. 47). Sul piano morfosintattico è tipica sia la collocazione dell'ausiliare alla fine della frase (come in "Montalbano sono" a p. 37 e "Sbirri siete?" a p. 46), sia l'uso del congiuntivo imperfetto al 
posto di quello presente, come si vede nella frase: “Taliasse ccà, dottore!” (p. 11), dove "Taliasse" significa guardasse e sta per "guardi".

Oltre ai termini dialettali, a caratterizzare la scrittura di Camilleri contribuiscono gli anacoluti, l'uso allusivo di certe interiezioni, le domande retoriche che restano senza risposta o che ottengono reticenti risposte monosillabiche, nonché i sottintesi che separano una scena dalla successiva. Rappresentando una forza particolare delle sue storie, il linguaggio contraddistingue i personaggi e diventa veicolo informativo del loro modus cogitandi: al burocratese degli impiegati si mescolano il parlato semplice e diretto dei poliziotti del commissariato di Vigàta, il dialetto della gente comune e il gergo allusivo dei mafiosi. Dando risalto alle varie sfumature e ambiguità linguistiche, in un mescolarsi di stili e registri, l'autore concretizza, pertanto, sulla pagina un ritmo teatrale e rende percepibile un aspetto della tragedialità dei siciliani, ossia della loro capacità di costruirsi maschere sempre diverse e di fare teatro, nella consapevolezza del valore pirandellianamente scompositivo racchiuso nel termine "Se..." (p. 206).

\section{La teatralità comportamentale}

Facendo rivivere nel testo i caratteri e le passioni dei suoi conterranei, Camilleri rievoca il genius loci ed eleva la sicilianità a grande metafora dell'umanità. In particolare, rappresentando i siciliani come attori in grado di fondere la vita e la scena, fa risaltare il loro essere persone e al tempo stesso personaggi, come si evince nel seguente passaggio: “«Oh Dio!» fece Montalbano, cominciando a recitare il secondo atto della sua parte, visto che la signora Clementina Vasile Cozzo la sua l'aveva fatta in modo perfetto" (p. 25). Montalbano, infatti, "quando ci si metteva sapeva essere uno strepitoso attore" (p. 22), in grado di portare avanti imperturbato "il suo teatro" (ibid.) e indossare i panni del tipico "teatrante" (SORGI 2000, p. 30). 
A differenza di Montalbano che sa assumere ruoli diversi in base all'occorrenza ed è in grado di esprimersi accompagnando a frasi sintetiche la mimica e la gestualità, alcuni personaggi assomigliano a dei pupi sia per il loro incedere meccanico, sia per il loro comportamento in balìa di forze superiori: ad esempio, il signor Pizzotta, direttore dell'albergo Jolly di Montelusa, luogo dell'omicidio di Michela Licalzi, “[...] si allontanò rigido che pareva un pupo di ligno in movimento" (p. 45); in un altro passaggio il dottor Lattes "[...] si voltò a taliare i sei agenti che calarono la testa in segno d'assenso: parsero pupi tirati dai fili”" (p. 118); invece l'avvocato Orazio Guttadauro "[...] è un pupo che la mafia gli tira i fili” (p. 131).

Al tono di poliedrica teatralità rintracciabile nella narrazione contribuisce il modo in cui sono caratterizzati i membri della squadra del commissariato di Vigàta, descritti con caratteristiche stereotipate, tic e manie che, senza infrangere le regole del giallo, conferiscono alla narrazione una notevole forza espressiva. Inoltre, nel tessuto narrativo de La voce del violino si riscontrano anche tocchi di vis comica che capovolgono la tragicità della situazione, come si legge nella parte successiva alla narrazione del suicidio di Guido Serravalle, il cui sangue aveva macchiato i vestiti del commissario:

\footnotetext{
Montalbano si dovette cangiare d'abito e di cammisa, cedendo alle istanze del direttore che volle prestargli cose sue. Negli abiti dell'omone ci stava due volte, con le mani perse dintra le maniche e i pantaloni a fisarmonica sopra la scarpe pareva il nano Bagonghi. [...] «Ti sei accorciato?» s’informò Mimì quando lo vide.

Per un pelo arriniscì a scansare il cazzotto di Montalbano che gli avrebbe rotto il naso. (p. 203)
}

Pseudonimo dei nani che lavorano nei circhi e nelle fiere, il termine "Bagonghi" può essere usato nella lingua parlata per definire in modo scherzoso una persona di statura non molto alta e, inserito in questo contesto, 
rappresenta quasi una caduta nel burlesco che procura al lettore una distanza critica dal racconto. ${ }^{13}$

Per cogliere la complessità della figura centrale del commissario occorre inserirla nel contesto siciliano di cui fa parte e tenere conto del fatto che è proprio lo sfondo che dà risalto alla sua figura, facendo emergere il suo carattere. Nel giallo in esame, Salvo Montalbano è un uomo di quarantacinque anni, solitario e orgoglioso, che non sa mentire alle persone che stima e che si sente costretto dalla fidanzata Livia a pensare al loro matrimonio e all'adozione di François; è "tradizionalista" (p. 83), dotato di senso dell'umorismo, colto e amante non solo della lettura, ma anche della buona cucina, tanto che, considerando il momento del pasto come rivestito quasi di sacralità, per non essere disturbato è solito staccare la spina del telefono. ${ }^{14}$ Montalbano non ama guidare la macchina e non ci sa fare né con il computer, né con i numeri. Ma ha una memoria fotografica eccezionale e tira le fila giuste, senza lasciarsi sfuggire nulla: è lui che avvia le indagini, facendo fare alla signora Vasile Cozzo la telefonata anonima alla questura per avvertire i superiori della presenza del cadavere di Michela Licalzi, ed è lui che ricostruisce lo svolgersi dei fatti, comprendendo che il suono del violino, diventando voce, è l'espediente che rivela l'elemento dissonante nel contesto,

\footnotetext{
${ }^{13}$ Riguardo alla vis comica in Camilleri cfr. Pistelli, 2003, p. 47: “[...] una prorompente e variegata vis comica che $[. .$.$] è in grado all'occorrenza di trasformarsi in strumento di conoscenza nei$ confronti della tragicomica natura del mondo siciliano. Una intelligente quanto dissacrante ironia questa di Camilleri $[. .$.$] basata sulla sua notevole abilità nell'utilizzare al meglio tutti i registri del$ comico (alternando battute triviali di matrice popolare, ad altre più sottili ma ugualmente taglienti espresse da Montalbano; introducendo gags tratte dal più classico dei repertori o persino personaggi ridotti quasi a macchiette di genere, come l'ingenuo e ridicolo Catarella)". Sul ruolo della risata in Camilleri e sugli elementi che la suscitano cfr. Palumbo, 2005, pp. 37-43.

${ }^{14}$ A riguardo si legge, infatti, che "per evitare che gli squilli gli turbassero la serenità di spirito indispensabile per gustare a fondo la salsetta, staccò la spina" (p. 36). Tra i numerosi passi in cui si fa riferimento a piatti tipici siciliani si vedano i seguenti: "una brioscia con la granita" (p. 15); "una grossa porzione di caponatina" (p. 97); "cannola [...] tetù, taralli, viscotti regina, mostazzoli di Palermo, dolci di Riposto, frutti di martorana e [...] una coloratissima cassata di cinque chili (p. 101). Il richiamo costante a ricette dell'arte culinaria siciliana può essere visto, più che come elemento folcloristico che sta sullo sfondo, come metafora per affermare l'importanza di una tradizione basata su principi semplici e genuini. Cfr. Pistelli, 2003, pp. 38-39.
} 
portandolo alla fulminante intuizione che il filo rosso attorno a cui si intreccia il doppio delitto è il concetto di scambio, non solo di violini. Sapendo cogliere i significati sottesi ai messaggi dei mafiosi, sa destreggiarsi nel gioco di mosse e contromosse condotto dai colleghi e riesce a risolvere l'omicidio del Di Blasi, delitto nel delitto Licalzi. Inoltre, fa da "regista occulto" (p. 120) dell'amico giornalista Nicolò Zito e fa smascherare i pupari, in possesso della prova con cui ricattare la polizia.

Eppure, nonostante la sicurezza e la determinazione con cui agisce, non mancano i momenti in cui si interroga sul proprio compito e sulla propria identità:

Passato il ponte, tirò dritto, non aveva gana di sentirsi dire un'altra volta macari da Anna che lui doveva assolutamente intervenire. A che titolo? Ecco a voi il cavaliere senza macchia e senza paura! Ecco a voi Robin Hood, Zorro e il giustiziere della notte tutti in una sola persona: Salvo Montalbano! (p. 116)

Oltre a farsi portavoce di un mondo siciliano tratteggiato tra finzione e verosimiglianza, il commissario esprime le ansie della società contemporanea, assumendo dei contorni caratteriali universali. ${ }^{15} \mathrm{Si}$ concorda, pertanto, con Palumbo nell'affermare che:

Montalbano porta in sé tutte le inquietudini e le solitudini dell'uomo europeo contemporaneo, cui è assimilato dalla forte apertura intellettuale e dalle dimensioni globalizzate delle problematiche sociali con cui si confronta. Ma possiede anche tutte le ricchezze e le energie dell'appartenenza a una cultura d'origine vivamente connotata. (PALUMBO 2005, p. 119)

\footnotetext{
${ }^{15}$ Per approfondire la visione che Camilleri ha del personaggio di Montalbano, tra gli altri, cfr. Lodato, 2002, pp. 375-414.
} 
Incarnando la figura del poliziotto schietto e capace, Montalbano non si preoccupa di nascondere la propria antipatia verso il nuovo questore ed è rispettato dai suoi collaboratori che conoscono il potenziale pericolo della sua "calma controllata" (p. 30). Difensore dello stato, onesto e incorruttibile, il commissario offre al lettore la possibilità di identificarsi in lui che, nella lotta contro le ingiustizie, "senza eroismi da sceriffo americano e con l'intelligenza acuta e spregiudicata e la densa umanità d'un buon siciliano, viene a capo delle cose" (CALABRÒ, 2004, p. 41).

\section{Conclusione}

Dall'analisi condotta appare evidente come ne La voce del violino la Sicilia appaia non come un'isola chiusa ai contatti con il mondo, né come metafora di tutto ciò che è negativo, bensì, al contrario, come spazio di incontro aperto a influssi dall'esterno ${ }^{16}$ e, soprattutto, come luogo mitico che incanta e offre protezione. È qui, infatti, che i Licalzi hanno fatto, per volontà della moglie, il loro viaggio di nozze e in quell'occasione la donna è rimasta affascinata dal posto: "Lei da sempre, senza conoscerla, mitizzava la Sicilia. Quando l'ha visitata, ne è rimasta incantata. Credo volesse crearsi un suo rifugio" (p. 90). Questa è la risposta che il dottor Licalzi dà a Montalbano alla domanda sul perché la moglie avesse voluto farsi costruire una villetta proprio sull’isola. La Sicilia, dunque, non assume i contorni di una terra tradizionalmente connotata come mafiosa, priva di sicurezza e largamente corrotta, ma è presentata da un'altra angolazione come una realtà credibile e potenzialmente vera nel suo intenso e contraddittorio fascino.

Dal testo emerge che sicilianità significa in primo luogo contraddizione: spiagge bellissime e colline aride, luoghi d'incontro e ampi spazi disabitati,

\footnotetext{
${ }^{16}$ Sul ruolo della Sicilia come unità distinta e come luogo aperto ai più diversi influssi afferma Meter, 2000, p. 152: "So ist Sizilien zwar eine distinkte historische und geographische Einheit, doch tendenziell offen für die diversesten Einflüsse und somit als Erfahrungsraum nicht nur zugänglich, sondern gar von spannungsreicher Attraktivität".
} 
superstrade e trazzere terremotate, onestà e correttezza, ma anche illegalità e corruzione. Sicilianità, però, non significa tout court criminalità mafiosa: alla perdita d'entusiasmo delle faide tra cosche mafiose si contrappone nettamente un delitto che ha come movente i debiti di gioco di un antiquario bolognese. Sicilianità significa ibridismo linguistico: alla lingua italiana si accompagna il dialetto, il quale conferisce ai discorsi una particolare forza espressiva e li colora di vivacità. Sicilianità significa teatralità, per cui viene messa in risalto la capacità di sdrammatizzare la realtà e di recitare una parte, accettando le regole del gioco.

Visto in questi termini, l'aspetto della sicilianità può costituire un punto di osservazione strategico da cui leggere e analizzare la vicenda. La presenza di diversi elementi e caratteri tradizionalmente peculiari della Sicilia e dei suoi abitanti, infatti, se, da un lato, avvicina il testo alla realtà e fa di questa sorta di realismo, come afferma Alewyn, ${ }^{17}$ non solo una questione stilistica, bensì quasi una componente fondamentale della struttura del genere letterario del giallo, dall'altro rende la finzione letteraria veramente plausibile, attribuendo all'universo siciliano di Montalbano una fisionomia attendibile. La sicilianità, dunque, nell'analisi di un testo ambientato nella cittadina fantastica di Vigàta, in terra di Sicilia, e scritto da un raccontastorie siciliano che ripercorre con il ricordo i luoghi vissuti, può rappresentare un valido punto di vista da cui avviare un'indagine narrativa, essendo tale punto di vista molto più che la visione di un punto.

\footnotetext{
${ }^{17}$ Alewyn, 1998, p. 69: "Der Realismus des Detektivromans ist nicht nur eine Frage des Stils, sondern auch ein fundamentales Element der Struktur".
} 


\section{Riferimenti bibliografici}

\section{Letteratura primaria}

CAMILLERI, Andrea. La forma dell'acqua. Palermo: Sellerio, 1994.

Il cane di terracotta. Palermo: Sellerio, 1996.

. Il ladro di merendine. Palermo: Sellerio, 1996.

. La voce del violino. Palermo: Sellerio, 1997.

. L'odore della notte. Palermo: Sellerio, 2001.

MACCHIAVELLI, Loriano. I sotterranei di Bologna. Milano: Mondadori, 2003.

SCERBANENCO, Giorgio. Traditori di tutti. Milano: Garzanti, 2003.

SCIASCIA, Leonardo. Il giorno della civetta. Milano: Adelphi, 1993.

\section{Letteratura secondaria}

CLAUSI, Maurizio; LEONE, Davide; LO BOCCHIARO, Giuseppe; PANCUCCI AMARÙ, Alice; RAGUSA, Daniela. I luoghi di Montalbano. Una guida. Palermo: Sellerio, 2007. 
BUTTITTA, Antonino (ed.). Il caso Camilleri. Letteratura e storia. Palermo: Sellerio, 2004.

ALEWYN, Richard. Anatomie des Detektivromans. In VOGT, Jochen (ed.). Der Kriminalroman. Poetik - Theorie - Geschichte. München: Fink, $1998,52-72$.

BONFIGLIO, Gianni. Siciliano - italiano. Piccolo vocabolario ad uso e consumo dei lettori di Camilleri e dei siciliani di mare. Roma: Fermento, 2002.

BONINA, Gianni. Il carico da undici. Le carte di Andrea Camilleri. Siena: Barbera, 2007.

CALABRÒ, Antonio. L’identità siciliana e la lezione di Camilleri. In BUTTITTA, Antonino (ed.). Il caso Camilleri. Letteratura e storia. Palermo: Sellerio, 2004, 31-41.

CAPECCHI, Giovanni. Andrea Camilleri. Firenze: Cadmo, 2000.

CERRATO, Mariantonia. L'alzata d'ingegno. Analisi sociolinguistica dei romanzi di Andrea Camilleri. Firenze: Cesati, 2012.

CROVI, Luca. Tutti i colori del giallo. Il giallo italiano da De Marchi a Scerbanenco a Camilleri. Venezia: Marsilio, 2002.

DEMONTIS, Simona. I colori della letteratura. Un'indagine sul caso Camilleri. Milano: Rizzoli, 2001. 
DI PAOLO, Paolo (ed). Ogni viaggio è un romanzo. Libri, partenze, arrivi.19 incontri con scrittori. Roma: Laterza, 2007.

FERLITA, Salvatore (ed.). La Sicilia di Andrea Camilleri. Tra Vigata e Montelusa. Palermo: Kalós, 2003.

LODATO, Saverio. La linea della palma. Saverio Lodato fa raccontare Andrea Camilleri. Milano: Rizzoli, 2002.

METER, Helmut. Andrea Camilleri oder der Kriminalroman als kulturelles Gedächtnis. In FELTEN, Hans; NELTING, David (ed.). ... una veritade ascosa sotto bella menzogna... Zur italienischen Erzählliteratur der Gegenwart. Frankfurt a. M. (et al.): Lang: 2000, 149-158.

PALUMBO, Ornella. L'incantesimo di Camilleri. Roma: Editori Riuniti, 2005.

PISTELLI, Maurizio. «Montalbano sono». Sulle tracce del più famoso commissario di polizia italiano. Firenze: Le Càriti, 2003.

PRIVITERA, Daniela. Il giallo siciliano da Sciascia a Camilleri (tra letteratura e multimedialità). Gela: Kronomedia, 2009.

RAMBELLI, Loris. Storia del “giallo” italiano. Milano: Garzanti, 1979.

SORGI, Marcello. La testa ci fa dire. Dialogo con Andrea Camilleri. Palermo: Sellerio, 2000. 
SORGI, Marcello. Camilleri e gli intellettuali siciliani. In BUTTITTA, Antonino (ed.): Il caso Camilleri. Letteratura e storia. Palermo: Sellerio, 2004, 42-47.

VITALE, Armando. Il mondo del commissario Montalbano. Considerazioni sulle opere di Andrea Camilleri. Caltanissetta: Terzo millennio, 2001. 